\title{
PRUEBA DE EFICIENCIA DÉBIL EN EL MERCADO ACCIONARIO COLOMBIANO*
}

Recibido: 30 de mayo de 2013 • Aprobado: 15 de mayo de 2014

\author{
César A. Ojeda Echeverri* \\ Elkin A. Castaño Vélez ${ }^{* * *}$
}

\section{RESUMEN}

Este trabajo prueba la hipótesis de eficiencia débil al comprobar la hipótesis de martingala en diferencias en los retornos del Índice General de la Bolsa de Valores de Colombia, IGBC. Se considera una estructura de dependencia condicional de primer orden mediante el modelo auto-regresivo fraccionalmente integrado de medias móviles, ARFIMA, y de segundo orden con el modelo hiperbólico asimétrico potencial autorregresivo condicionalmente heterocedástico, HYAPARCH, el cual captura todos los hechos estilizados encontrados en la investigación empírica. Los resultados rechazan la hipótesis de eficiencia débil al mostrar que el proceso generador de los retornos parece obedecer a un modelo auto-regresivo fraccionalmente integrado, arfi, en media condicional y a un hiperbólico asimétrico autorregresivo condicionalmente heterocedástico, HYAGARCH, en varianza condicional.

\section{PALABRAS CLAVE}

IGBC, eficiencia débil, volatilidad, ARFIMA, HYAPARCH

\section{CLASIFICACIÓN JEL}

C01, C22, C51, C58, G14

\section{CONTENIDO}

Introducción; 1. Modelo de martingala en diferencias; 2. Análisis estadístico preliminar; 3. Pruebas de memoria larga; 4. Modelo propuesto para el retorno del IGBC; 5. Conclusiones y recomendaciones; Bibliografía; Anexo.

* Artículo de investigación producto del trabajo de tesis de Maestría en Ciencias Estadística desarrollada por César Ojeda, titulada "Una prueba de la eficiencia débil en el mercado accionario Colombiano", bajo la asesoría del Profesor Elkin Castaño Vélez de la Escuela de Estadística, Universidad Nacional de Colombia, Medellín, Colombia. Periodo de ejecución: 2011-2013.

** Estadístico de la Universidad del Valle, Cali, Colombia. Magíster en Ciencias Estadística de la Universidad Nacional de Colombia, Medellín, Colombia. Profesor de la Escuela de Estadística de la Universidad del Valle, Cali, Colombia. Correo electrónico: cesar.ojeda@correounivalle.edu.co.

Estadístico, Universidad de Medellín, Colombia. Magíster en Ciencias Estadística, Instituto de Matemática Pura y Aplicada, IMPA, Río de Janeiro, Brasil. Magíster en Ciencias Estadísticas, Universidad Nacional de Colombia, Medellín, Colombia. Profesor de la Facultad de Ciencias de la Universidad Nacional de Colombia, Medellín, Colombia. Profesor de la Facultad de Economía de la Universidad de Antioquía, Medellín, Colombia. Director del Centro de Estadística Aplicada a Estudios Económicos (CEAES), Universidad Nacional de Colombia, Medellín, Colombia. Correo electrónico: elkincv@gmail.com. 


\section{WEAK EFFICIENCY TEST IN THE COLOMBIAN STOCK MARKET ABSTRACT}

This paper proves the weak efficiency hypothesis when proving the maritingala hypothesis on return differences for the General Index of the Colombian Stock Exchange (IGBC) . A first order conditional dependency structure by using the Autoregressive Fractionally Integrated Moving Averages model ARFIMA, and on second order with the Hyperbolical Asymmetric Autoregressive Potential Conditionally Heteroscedastic model, HYAPARCH, which captures all the stylized facts in the empiric research is considered. The results reject the weak efficiency hypothesis when showing that the returns generation process seems to obey to and Autoregressive Fractionally Integrated model ARFI in conditional average and a Hyperbolical Asymmetric Conditionally Heteroscedastic model, $\mathrm{HYAGARCH}$, in conditional variance.

\section{KEY WORDS}

IGBC, Weak efficiency, Volatility, ARFIMA, HYAPARCH

\section{JEL CLASSIFICATION}

C01, C22, C51, C58, G14

\section{CONTENT}

Introduction; 1 . Martigala difference model; 2. Preliminary statistical analysis; 3. Long term memory tests; 4 . Model proposed for IGBC return; 5 . Conclusions and recommendations; Bibliography.

\section{PROVA DE EFICIÊNCIA DÉBIL NO MERCADO ACIONÁRIO COLOMBIANO RESUMO}

Este trabalho prova a hipótese de eficiência débil ao comprovar a hipótese de Gamarra em diferenças nas voltas do Índice Geral da Bolsa de Valores da Colômbia, IGBC. Considera-se uma estrutura de dependência condicional de primeiro ordem mediante o modelo Auto-regressivo Fraccionalmente Integrado de Médias Móveis, ARFIMA, e de segundo ordem com o modelo Hiperbólico Assimétrico Potencial Auto-regressivo Condicionalmente Heterocedástico, HYAPARCH, o qual captura todos os fatos estilizados encontrados na investigação empírica. Os resultados recusam a hipótese de eficiência débil ao mostrar que o processo gerador das voltas parece obedecer a um modelo Auto-regressivo Fraccionado Integrado, ARFI, em média condicional e a um Hiperbólico Assimétrico Auto-regressivo Condicionalmente Heterocedástico, HYAGARCH, em variância condicional.

\section{PALAVRAS CHAVES}

IGBC, Eficiência débil, Volatilidade, ARFIMA, HYAPARCH.

\section{CLASSIFICAÇÃO JEL}

\section{CONTEÚDO}

C01, C22, C51, C58, G14.

Introdução; 1. Modelo de Gamarra em diferenças; 2. Análise estatística preliminar; 3. Provas de cor longa; 4. Modelo proposto para a volta do IGBC; 5 . Conclusões e recomendações; Bibliografia; Anexo. 


\section{INTRODUCCIÓN}

De acuerdo con Dimson y Mussavian (2000), el concepto de eficiencia es clave en finanzas y es usado para describir un mercado en el cual toda la información relevante y disponible se encuentra reflejada en los precios de los activos financieros. Roberts (1967) distingue tres conjuntos de información relevante: la historia de los precios de los activos, la información pública disponible y la información privada. A partir de estos tres conjuntos se establecen tres formas de eficiencias: la débil, la semifuerte y la fuerte. Bajo la forma débil de eficiencia, se dice que los precios actuales de los activos reflejan en su totalidad la información contenida en los movimientos previos de los precios, es decir, con base en los precios históricos es imposible predecir los movimientos futuros de los precios ni, por tanto, crear estrategias exitosas de especulación.

La idea de falta de predictibilidad en los precios de los activos financieros ha sido referida como la hipótesis de caminata aleatoria, según Escanciano y Lobato (2009). Campbell, Lo y MacKinlay (1997) distinguen tres tipos de caminatas aleatorias de acuerdo con la estructura de dependencia de los incrementos en los precios: la caminata aleatoria 1 corresponde a incrementos independientes; la caminata aleatoria 2 corresponde a incrementos independientes en media, y la caminata aleatoria 3 corresponde a incrementos incorrelacionados.

En particular, la caminata aleatoria 2 cumple con la propiedad de martingala, lo cual significa que el mejor pronóstico del precio de mañana del activo financiero es el precio de hoy. En términos de los retornos (incrementos), esta propiedad se conoce como martingala en diferencias, esto es, los retornos de un activo deben conformar un juego justo. LeRoy (1989, pp. 1588-1589) menciona que "... el modelo de martingala captura la "esencia" del modelo de caminata aleatoria", y "... constituye un auténtico modelo económico para los precios de los activos financieros".

Samuelson (1965) muestra cómo, desde el punto de vista de la eficiencia informacional, los precios deben ser impredecibles, y deduce que los retornos de un activo deben ser un juego justo. Mandelbrot (1966) propone el modelo de martingala para los precios accionarios, en donde los cambios sucesivos de precios son dependientes, y la naturaleza de dicha dependencia hace que esta no pueda ser usada para aumentar las ganancias esperadas. Fama (1970, p. 383) desarrolla esta idea al definir que "un mercado en el que los precios reflejan plenamente toda la información disponible es eficiente".

Bajo la idea de Andreou, Pittis y Spanos(2001), y LeRoy (1989), el objetivo principal de este trabajo consiste en probar la hipótesis de eficiencia débil al comprobar la 
hipótesis de martingala en diferencias en los retornos accionarios del Indice General de la Bolsa de Colombia (IGBC), mediante la verificación de la existencia de posibles efectos medios condicionales significantes al tener en cuenta una posible estructura de dependencia de mayor orden. La existencia de efectos medios condicionales significantes en los retornos accionarios del activo sugiere el rechazo de la hipótesis de eficiencia débil. De acuerdo con Fama (1970), dicha prueba puede ser realizada bajo supuestos como la inexistencia de costos de transacción, neutralidad ante el riesgo por parte de los participantes del mercado, y que toda la información disponible pueda obtenerse sin costo por todos los participantes.

Para la realización de este trabajo se cuenta con precios diarios desde el 3 de julio de 2001 (fecha de inicio de actividades para la Bolsa de Valores de Colombia) hasta el 3 de marzo de 2011, para un total de 2360 observaciones. Estos datos fueron suministrados por la Bolsa de Valores de Colombia (regional Medellín).

Gran parte de los trabajos existentes sobre la eficiencia del mercado accionario colombiano enfocan la eficiencia débil desde el punto de vista de la caminata aleatoria 1. Por ejemplo, trabajos como los de Worthington y Higgs (2003), Maya y Torres (2004, 2005), Méndez (2009) usan pruebas de raíces unitarias, pruebas de rachas, pruebas de razón de varianzas, entre otras, para probar dicha hipótesis. Sin embargo, la noción de la caminata aleatoria 1 es rechazada en los datos financieros por muchas razones, entre las cuales, la más importante es la dependencia temporal de segundo orden de los incrementos de los precios como lo presenta Engle (1982).

En términos de la hipótesis de martingala en diferencias para los retornos del IGBC, algunos trabajos existentes como Pérez y Fernández (2006), Montenegro (2007), Rivera (2009),enfocan su metodología en modelos generalizados auto-regresivos condicionalmente heterocedásticos (GARCH) para encontrar estructuras de dependencia en el proceso de media condicional, y así valorar dicha hipótesis. Aunque estos trabajos encuentran algunas estructuras de dependencia significativas, los autores mencionan que es probable que al utilizar metodologías posteriores a los GARCH, se encuentren valores más ajustados a la realidad del mercado. Pérez (2009) profundiza en este aspecto y utiliza el modelo auto-regresivo y de medias móviles fraccionalmente integrado (ARFIMA) en media condicional y el modelo generalizado auto-regresivo condicionalmente heterocedástico y fraccionalmente integrado (FIGARCH) para la volatilidad condicional con el objetivo de evaluar la existencia de memoria larga como una prueba de la eficiencia débil. El autor menciona que la existencia de memoria larga en cualquiera de estos dos modelos va en contra de dicha hipótesis. Los resultados muestran que existe un grado de persistencia en la serie, lo que indica que el mercado accionario colombiano no es eficiente. Sin 
embargo, Davidson (2004) muestra cómo el modelo FIGARCH no es estacionario, y su memoria incrementa cuando el parámetro de diferenciación fraccional tiende a cero, contrario al rol que ejerce este mismo parámetro en el modelo fraccionalmente integrado en media condicional. Por tanto, la caracterización del modelo FIGARCH como un caso intermedio entre el modelo GARCH y el modelo integrado generalizado auto-regresivo condicionalmente heterocedástico (IGARCH), sería poco adecuado.

En este trabajo se emplea el modelo ARFIMA en media condicional, y el modelo hiperbólico-asimétrico-potencial generalizado auto-regresivo condicionalmente heterocedástico (HYAPARCH) en volatilidad condicional, el cual permite capturar un conjunto mayor de posibles dinámicas, en comparación con los modelos anteriormente mencionados, y así obtener valores más ajustados a la realidad del mercado. En particular, este modelo contempla todas las características (hechos estilizados) encontradas en las investigaciones empíricas: volatilidad por agrupamiento, leptocurtosis, asimetría y memoria a largo plazo, tal y como lo menciona Schoffer (2003).

El artículo se desarrolla en el siguiente orden: en la primera parte, se define el concepto de martingala, y martingala en diferencias, y su posible extensión al considerar momentos condicionales de mayor orden; en la segunda parte, se describe la información utilizada y se realiza el análisis estadístico preliminar de la misma; en la tercera parte, se realizan algunas pruebas de memoria larga sobre la serie analizada; en la cuarta parte, se presentan la especificación y la estimación del modelo propuesto, y se comprueba la hipótesis de martingala en diferencias para considerar la hipótesis de eficiencia débil; finalmente, se exponen las conclusiones y recomendaciones.

\section{MODELO DE MARTINGALA EN DIFERENCIAS}

Ya que los precios de los activos financieros no son estacionarios, desde un punto de vista técnico, es más conveniente manejar los retornos del activo, y en lugar de probar que los precios siguen una martingala, es más común probar que los retornos siguen una sucesión de martingala en diferencias. Una sucesión de martingala en diferencias no tiene ninguna dependencia en media, condicionada a su propio pasado, lo que implica que sea impredecible a partir de su propio pasado. Al respecto, LeRoy (1989, p. 1590) menciona que "la martingala en diferencias descarta cualquier dependencia de la esperanza condicional de los retornos sobre su propio pasado, mientras que la caminata aleatoria 1 descarta, además de esto, la dependencia que implican los momentos condicionales de mayor orden". 
Andreou, Pittis y Spanos (2001, p. 204) y LeRoy (1989, p. 1590) mencionan que "la prueba empírica más directa sobre la idoneidad de la hipótesis de martingala en diferencias asciende a determinar si existen efectos medios condicionales significantes".

Sea $\mathrm{P}_{t}$ el precio observado en el tiempo $t$ de un activo financiero. Se dice que $\mathrm{P}_{t}$ cumple con la propiedad de martingala si para todo $t$ se cumple que:

$$
\mathrm{E}\left(\mathrm{P}_{t} \mid \sigma\left(\mathrm{P}_{t-1}, \ldots, \mathrm{P}_{1}\right)\right)=\mathrm{P}_{t-1}
$$

donde $\sigma\left(\mathrm{P}_{t-1}, \ldots, \mathrm{P}_{1}\right)$ es el conjunto de información disponible en el tiempo $t-1$.

Además, sea $r_{t}$ el retorno observado en el tiempo $t$ de un activo financiero. Se dice que $r_{t}$ cumple con la propiedad de martingala en diferencias, si para todo $t$ se cumple que:

$$
\mathrm{E}\left(r_{t} \mid \sigma\left(r_{t-1}, \ldots, r_{1}\right)\right)=0
$$

donde $\sigma\left(r_{t-1}, \ldots, r_{1}\right)$ es el conjunto de información disponible en el tiempo $t-1$.

La estructura probabilística de un proceso que cumple con la propiedad de martingala en diferencias es mucho más débil que la de un proceso Independiente e idénticamente distribuido (IID), con momentos hasta de orden 2. El supuesto IID puede ser expresado como:

$$
f\left(r_{t} \mid r_{t-1}, \ldots, r_{1} ; \theta\right)=f\left(r_{t} ; \theta\right) \text { para todo } t .
$$

donde $f$ representa una función de densidad de probabilidad dada y $\theta$ representa sus parámetros. En términos de los momentos condicionales (al suponer que existen), (1) puede ser expresado de la siguiente forma:

$$
\mathrm{E}\left(r_{t}^{k} \mid \sigma\left(r_{t-1}, \ldots, r_{1}\right)\right)=E\left(r_{t}^{k}\right), \text { para } k=1,2, \ldots, \mathrm{y} \text { para todo } t .
$$

Este punto fue formulado por Mandelbrot (1966, p. 244), cuando introduce el modelo de martingala para los precios accionarios. Él afirma de manera explícita:

$[\ldots]$ sea $t, t+\mathrm{T}$ y $\mathbf{t}_{\mathrm{i}}^{0}$ un instante en tiempo presente, un instante en tiempo futuro, y un conjunto de instantes pasados, respectivamente. $Z(t)$ es martingala si se cumple que $E\left[Z(t+T)\right.$, dado los valores de $Z(t)$ y para todo $\left.Z\left(t_{i}^{0}\right)\right]=Z(t)$...cabe destacar que la distribución de $\mathbf{Z}(\mathbf{t}+\mathbf{T})$, condicionado por valores conocidos de $\mathbf{Z}(\mathrm{t})$ y de $\mathbf{Z}\left(\mathrm{t}_{\mathrm{i}}^{0}\right)$ pueden depender de los valores pasados de $\mathbf{Z}\left(\mathrm{t}_{\mathrm{i}}^{0}\right)$; solo la esperanza es la que no es afectada por $\mathbf{Z}\left(\mathbf{t}_{\mathrm{i}}^{0}\right)$. 
Con esta idea, Andreou, Pittis y Spanos(2001) muestran cómo se puede extender un proceso de martingala en diferencias al tener en cuenta momentos condicionales de mayor orden, bajo el supuesto de que existen. En particular, dicha dependencia puede ser establecida como:

$$
\left.\begin{array}{c}
\mathrm{E}\left(r_{t} \mid r_{t-1}, \ldots, r_{1}\right)=0, \\
\mathrm{E}\left(r_{t}^{k} \mid r_{t-1}, \ldots, r_{1}\right)=g_{k}\left(r_{t-1}, r_{t-2}, \ldots, r_{1}\right), \text { para } k=2,3, \ldots,
\end{array}\right\} \text { para todo } t .
$$

Así, por ejemplo, un proceso de martingala en diferencias de segundo orden se define como (3) cuando $k=2$. Sobre este punto, Mandelbrot (1971, p. 226-227) menciona:

[...]Sea $\mathrm{P}(\mathrm{t})$ el precio en el tiempo $\mathrm{t}$, tal que $\mathrm{P}(\mathrm{t}+\mathrm{S})$ - $\mathrm{P}(\mathrm{t})$ es el cambio aleatorio del precio entre el tiempo presente t y el tiempo futuro $t+S$...el modelo de caminata aleatoria afirma que la distribución de probabilidad de $\mathrm{P}(\mathrm{t}+\mathrm{S})-\mathrm{P}(\mathrm{t})$ es independiente del precio actual $\mathrm{P}(\mathrm{t})$ y de todos los precios pasados...el modelo de martingala es menos exigente, suponiendo solo que el valor esperado condicional de $\mathrm{P}(\mathrm{t}+\mathrm{S})-\mathrm{P}(\mathrm{t})$ sobre el precio presente y/o varios precios pasados, se anula. Esto simplemente expresa que no existe una política de compra y venta que tenga un retorno esperado mayor que el retorno promedio del mercado. Por otra parte, el modelo de martingala permite que la distribución actual de $\mathrm{P}(\mathrm{t}+\mathrm{S})-\mathrm{P}(\mathrm{t})$ dependa de los precios pasados y presentes, y por tanto no niega que los precios pasados o presentes puedan servir en la selección de portafolios de diferentes niveles de riesgo deseados.

\section{ANÁLISIS ESTADÍSTICO PRELIMINAR}

La base de datos ${ }^{1}$ utilizada para este trabajo fue suministrada por la Bolsa de Valores de Colombia, regional Medellín, la cual contiene los precios de cierre diarios del IGBC desde el 3 de julio de 2001 hasta el 3 de marzo de 2011. Para la realización de los cálculos descriptivos y la representación gráfica es usando el programa ${ }^{2}$ estadístico $\mathrm{R}$ de R Core Team (2012).

Según Spanos (1995), la primera etapa en la comprobación de la hipótesis de eficiencia de mercado es la postulación de un adecuado modelo estadístico en el que la hipótesis de martingala en diferencias pueda ser probada. La elección de dicho modelo depende en gran parte de la estructura probabilística de los datos. Es así como esta sección intenta describir tal estructura probabilística en los retornos del IGBC.

\footnotetext{
Los lectores interesados en obtener los datos utilizados en este estudio pueden solicitarlos vía correo electrónico cesar.ojeda@correounivalle.edu.co.

2 Para una descripción de los paquetes utilizados, ver anexo A.
} 
Para iniciar, se calculan los retornos porcentuales continuamente compuestos o log-retornos como:

$$
r_{t}=\left(\log \mathrm{P}_{t}-\log \mathrm{P}_{t-1}\right) \times 100
$$

donde $\mathrm{P}_{t}$ es el precio del índice en el día $t$, y $\mathrm{P}_{t-1}$ es el precio del índice en el día anterior. En el gráfico 1, se presenta la serie de los precios del índice, sus retornos y sus correspondientes transformaciones (retornos al cuadrado y en valor absoluto).

Gráfico 1. Serie de precios del IGBC $\left(\operatorname{IGBC}_{t}\right)$, retornos porcentuales $\left(r_{t}\right)$, retornos porcentuales al cuadrado $\left(r_{t}^{2}\right)$ y en valor absoluto $\left(\left|r_{t}\right|\right)$.

$\mathrm{IGBC}_{\mathrm{t}}$

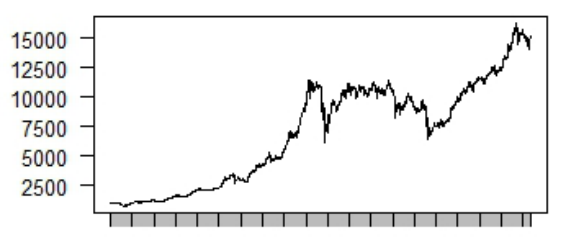

jul 032001 jul 012004 jul 032007 jul 012010

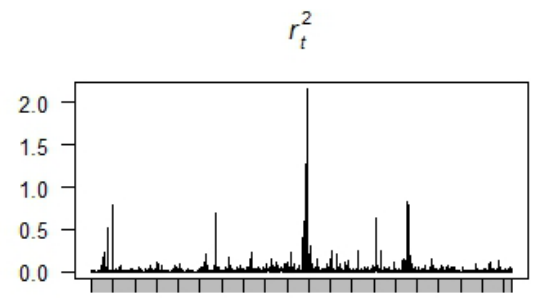

jul 032001 jul 012004 jul 032007 jul 012010 $r_{t}$

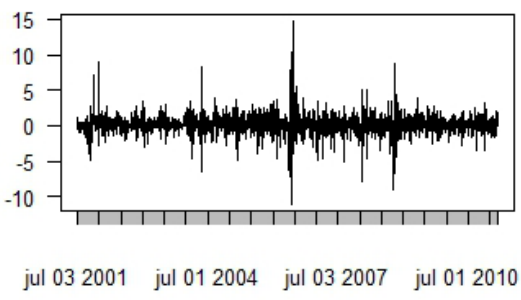

$\left|r_{t}\right|$

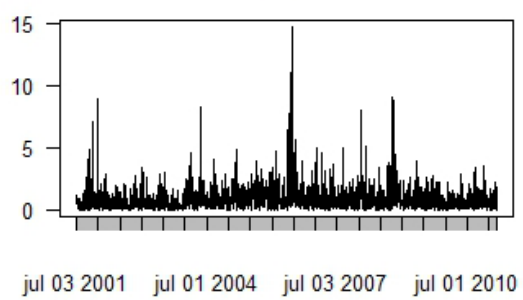

Fuente: elaboración propia

La inspección visual de la serie del IGBC parece indicar no estacionaridad, mientras que la de los retornos indica lo contrario, por lo menos en media. Además, para explorar una posible dependencia temporal no lineal en los retornos, se presentan los retornos al cuadrado y los valores absolutos de los mismos, lo cual indica que la varianza en el mercado es cambiante en el tiempo, y sugieren la existencia de volatilidad por agrupamiento, es decir, grandes (o pequeños) cambios en los precios tienden a estar agrupados. Por otro lado, la serie de los retornos parece tener una distribución incondicional leptocúrtica, ya que se observa una gran concentración alrededor de la media y un gran número de outliers relativo a la distribución normal. 
En términos de estos outliers, se observa que existen dos eventos muy relevantes en donde la volatilidad (variación) de los precios es muy alta. Estos eventos corresponden, por un lado, a la más dura caída de la historia de la bolsa colombiana en junio de 2006 la cual se presentó en medio del nerviosismo de los inversionistas quienes se apresuraron a vender sus acciones ante el rumor de un alza en los intereses de la Reserva Federal de Estados Unidos tal y como lo menciona Baquero (2006). Por otro lado, y de acuerdo con Ocampo (2009), la repercusión de la crisis financiera a mediados de octubre de 2008.

En la tabla 1 se presenta el resumen estadístico para los retornos porcentuales (en adelante solo retornos) del IGBC. Se observa que el retorno medio incondicional para el período bajo análisis es del $0.12 \%$, con una desviación estándar incondicional de $1.44 \%$. El retorno máximo observado para este período es de $14.69 \%$, mientras que el mínimo es de $-11.05 \%$. Además, se observa que más del $50 \%$ de los retornos es positivo. Por otro lado, se observa que existe un notable exceso de curtosis ${ }^{3}$ con respecto a la distribución normal, mientras que el coeficiente de asimetría es negativo y pequeño; en consecuencia, el contraste de normalidad incondicional JB de Jarque y Bera (1987) rechaza la hipótesis nula de normalidad. La prueba LB de Ljung y Box (1978) evidencia, además, una posible correlación serial significativa al rezago 20, tanto en los retornos como en sus cuadrados representados por la prueba ML de McLeod y Li (1983) lo que sugiere la posible necesidad de modelar tanto la media como la volatilidad condicional del proceso. Esto también es confirmado por la prueba de efectos ARCH de Engle (1982), la cual evidencia una posible existencia de heterocedasticidad condicional.

Tabla 1.Resumen descriptivo para el retorno del IGBC

\begin{tabular}{|c|c|c|c|c|c|}
\hline Estadísticas & $r_{t}$ & Estadísticas & $r_{t}$ & Estadísticas & $r_{t}$ \\
\hline Media & 0,12 & Asimetría & $-0,23$ & JB & 0,00 \\
\hline Mediana & 0,15 & Ex. Curtosis & 12,55 & LB(20) & 0,00 \\
\hline Máximo & 14,69 & ler Cuartil & $-0,48$ & $\operatorname{ML}(20)$ & 0,00 \\
\hline Mínimo & $-11,05$ & 3er Cuartil & 0,79 & $\mathrm{ARCH}(20)$ & 0,00 \\
\hline Des. Est. & 1,44 & & & & \\
\hline
\end{tabular}

Fuente: elaboración propia

En el panel (a) del gráfico 2 se presenta el histograma junto con el estimador no paramétrico de la función de densidad de probabilidad de la serie de los retornos, tal y como se muestra en Silverman(1986), los cuales muestran de nuevo el fuerte

3 El exceso de curtosis se define como el coeficiente de curtosis menos tres. 
exceso de curtosis en comparación con la función de densidad bajo la hipótesis de normalidad. Además, se presenta el comportamiento de estas estimaciones en las colas, tanto a la izquierda (b), como a la derecha (c). Estas estimaciones parecen indicar que la función de densidad incondicional de los retornos posee colas pesadas. Esto evidencia que grandes retornos, tanto positivos como negativos, ocurren con mayor frecuencia en comparación con lo esperado bajo normalidad.

Gráfico 2. (a) Histograma y densidad kernel estimada para los retornos del IGBC. (b) y (c) estimaciones en las colas a izquierda y derecha, respectivamente.

(a)

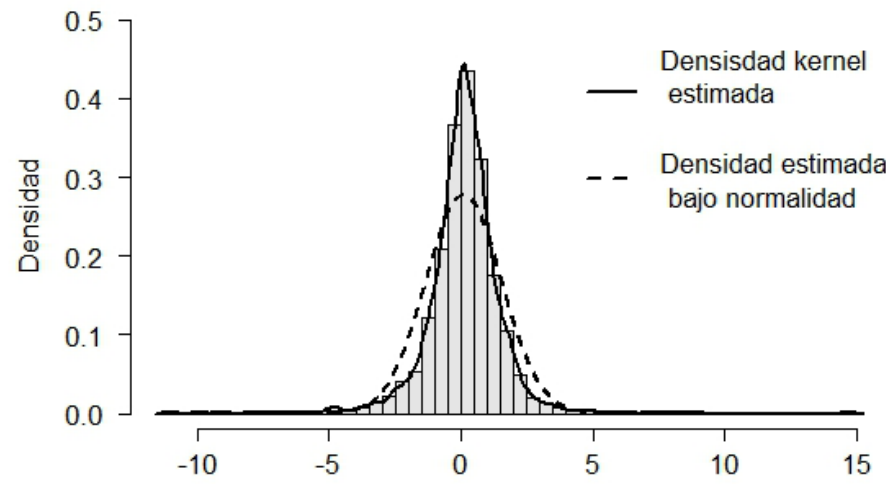

(b)

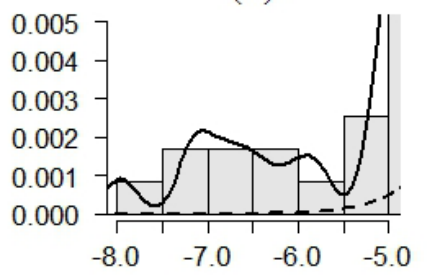

(c)

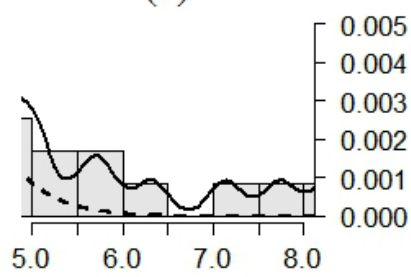

Fuente: elaboración propia

Para examinar características probabilísticas adicionales de los retornos, la densidad no paramétrica bivariada $f\left(r_{t}, r_{t-1}\right.$; È $)$ es estimada bajo la metodología de Bowman y Azzalini (1997). Esta densidad se observa en el gráfico 3, y con propósitos de comparación se presenta el gráfico 4 y el gráfico 5, los cuales muestran la función de densidad bajo la hipótesis de normalidad bivariada y bajo la hipótesis t-student bivariada con 9 grados de libertad, respectivamente. El gráfico 3 indica que la densidad estimada es unimodal, simétrica y leptocúrtica al exhibir un gran pico y "relativas" colas pesadas. Una evidencia adicional de la simetría es proporcionada por la estimación de los contornos presentados en los gráficos. Aunque no se presentan en este trabajo, las estimaciones de densidad bivariadas para otros rezagos, $r_{t-2}$ y $r_{t-3}$ presentan evidencia muy similar. Además, se observa una muy 
marcada similitud entre la estimación de la función de densidad bivariada de los retornos (gráfico 3) y la función de densidad estimada bajo la hipótesis de distribución t-student bivariada (gráfico 5).

Gráfico 3. Estimación de la función de densidad bivariada del IGBC.

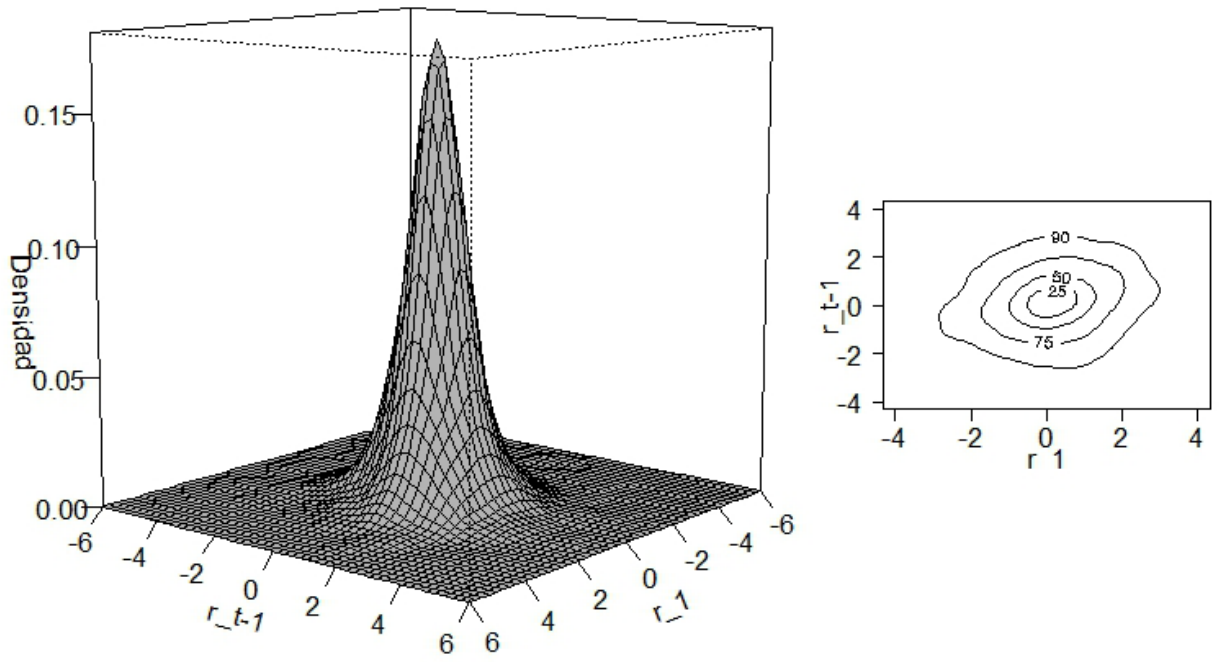

Fuente: elaboración propia

Gráfico 4. Distribución bivariada bajo normalidad
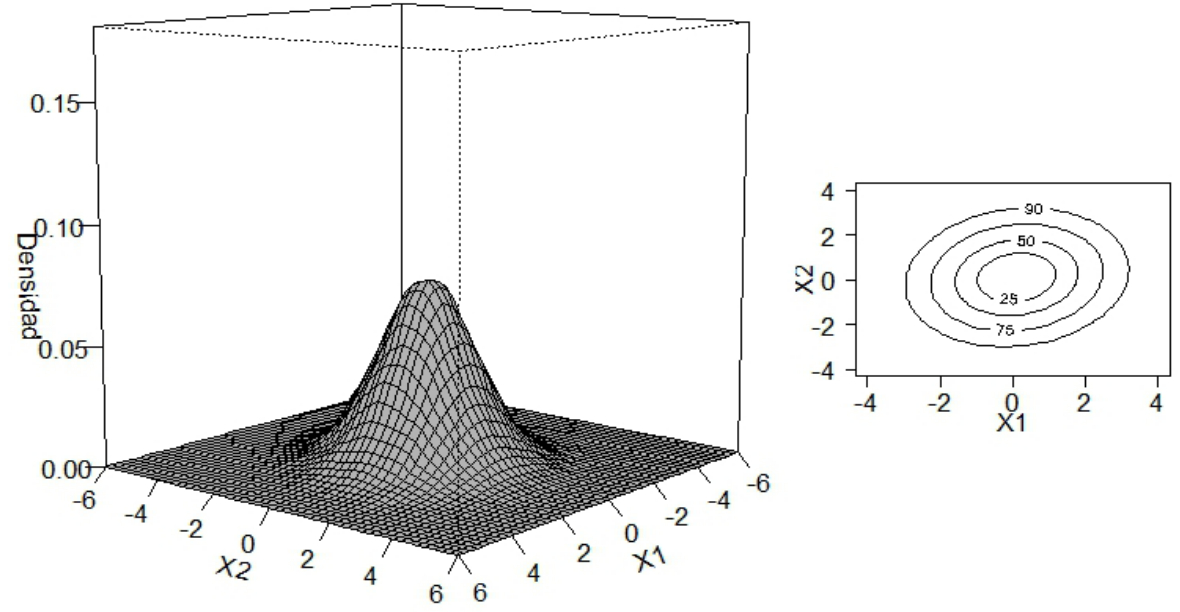

Fuente: elaboración propia 
Gráfico 5. Distribución bivariada bajo t-Student con v $=9$

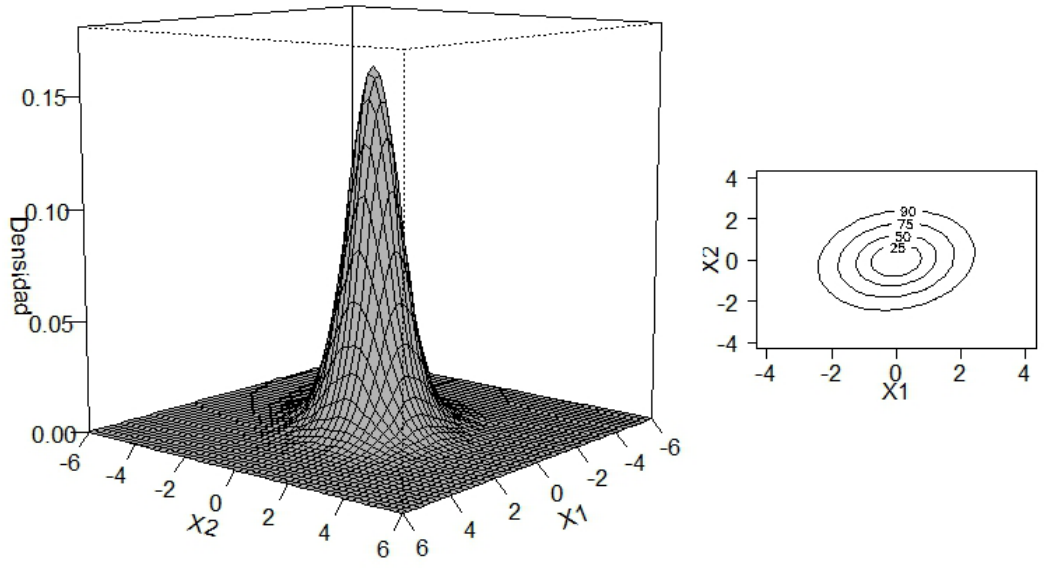

Fuente: elaboración propia

Gráfico 6. Correlogramas para el retorno del IGBC y sus transformaciones
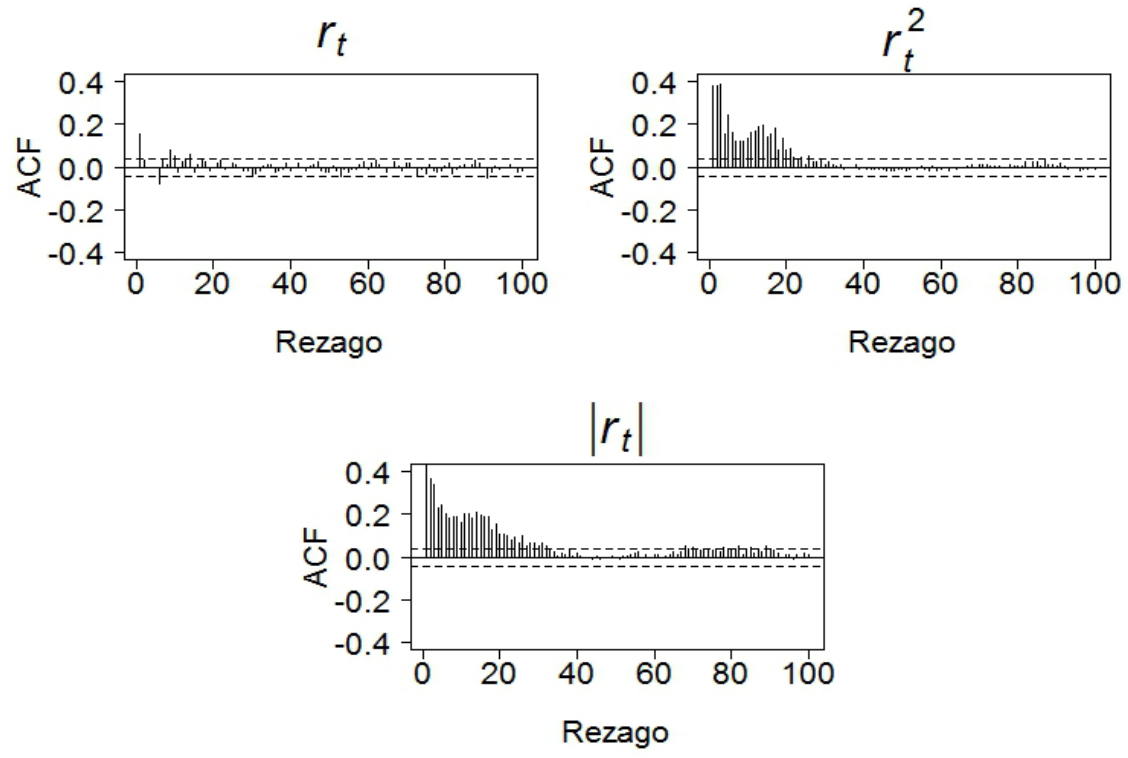

Fuente: elaboración propia

Por otro lado, dada la evidencia empírica de existencia de memoria a largo plazo en las series financieras, tal y como se muestra en Baillie (1996), Ding y Granger (1993), Ding, Granger y Engle (1996) y Lo (1991), se procede a investigar su presencia en los retornos del IGBC, así como en sus cuadrados y sus valores absolutos. 
Se emplean los correlogramas muestrales para los cien primeros rezagos, los cuales se presentan en el gráfico 6. Para los retornos al cuadrado y los valores absolutos, se confirma de manera visual la existencia de memoria larga al presentar patrones de decrecimiento hiperbólico congruentes con un comportamiento de persistencia en el proceso de volatilidad. Sin embargo, al observar el correlograma para los retornos del IGBC parece no existir evidencia a favor de larga memoria.

\section{PRUEBAS DE MEMORIA LARGA}

Dada la posible existencia de memoria larga en el proceso de volatilidad sugerida por los resultados presentados en la sección anterior, a continuación se realiza una serie de test encaminados a brindar una mayor claridad acerca de dicha hipótesis. Willinger, Taqqu y Teverovsky (1999) recomiendan no usar una única prueba para identificar memoria larga en un conjunto de datos, y promueven el uso de diversos métodos estadísticos, tanto gráficos como analíticos.

Las pruebas realizadas son: la prueba no paramétrica de Robinson y Lobato (1998); la prueba KPSS ${ }^{4}$ modificada de Lee y Schmidt (1996); la prueba del rango reescalado modificado de Lo (1991), y la prueba HML de Harris, McCabe y Leybourne (2008). La estimación del parámetro de diferenciación fraccional d es realizada mediante el estimador de Robinson (1995). En todas las pruebas, la hipótesis nula a probar es que el proceso $z_{t}$ es I $(0)$, es decir, el proceso es de memoria corta; la hipótesis alternativa es que el proceso $z_{t}$ es de memoria larga, esto es, el proceso es I $(d)$ con $d>0$.

Además, en la realización de las pruebas se utiliza el procedimiento heterocedástico y auto-regresivamente consistente, HAC, de Newey y West (1987) para la estimación de la matriz de covarianzas, el cual realiza una estimación consistente en presencia de heterocedasticidad y auto-correlación. También, se utiliza el kernel espectral cuadrático (OS) para la estimación HAC el cual, según Gil-Alana (2003), bajo estudios de Monte Carlo produce buenos resultados. Por último, el método plug-in de Newey y West (1994) es usado para la selección de los parámetros de truncamiento. Todas estas pruebas son realizadas en el programa Time Series Modelling (TSM) de Davidson (2011), el cual trabaja bajo el sistema OX de Doornik (2007).

$4 \quad$ Prueba de Kwiatkowski, Phillips, Smichdt y Shin (KPSS). 
Tabla 2. Pruebas de memoria larga para el retorno del IGBC y sus transformaciones

\begin{tabular}{lccc}
\hline \multicolumn{4}{c}{ Pruebas para $r_{t}$, Robinson's $d=0,09$} \\
\hline \multicolumn{1}{r}{ Pruebas I(0) } & Estadístico & Valor $p$ & Otros* $^{*}$ \\
\hline Robinson-Lobato & 0,54 & 0,30 & $m=292$ \\
KPSS & 0,34 & $\approx 1$ & QS, $6 w=3$, N-W plug-in \\
Lo's RS & 1,94 & $<0,03$ & QS, $6 w=3$, N-W plug-in \\
HML & 0,13 & 0,45 & $c=1, \mathrm{~L}=0,66$ \\
\hline
\end{tabular}

Pruebas para $r_{t}^{2}$, Robinson's $d=0,26$

\begin{tabular}{lccc}
\hline \multicolumn{1}{r}{ Pruebas I(0) } & Estadístico & Valor $p$ & Otros* \\
\hline Robinson-Lobato & 8,35 & 0 & $m=160$ \\
KPSS & 0,40 & $<0,1$ & OS, $6 w=6, \mathrm{~N}$-W plug-in \\
Lo's RS & 1,97 & $<0,03$ & OS, $6 w=6, \mathrm{~N}-\mathrm{W}$ plug-in \\
HML & 3,08 & 0,00 & $c=1, \mathrm{~L}=0,66$ \\
\hline
\end{tabular}

Pruebas para $\left|r_{t}\right|$, Robinson's $d=0,31$

\begin{tabular}{lccc}
\hline \multicolumn{1}{c}{ Pruebas I(0) } & Estadístico & Valor $p$ & Otros* \\
\hline Robinson-Lobato & 11,17 & 0 & $m=143$ \\
KPSS & 0,87 & $<0,01$ & QS, $6 w=6$, N-W plug-in \\
Lo's RS & 3,08 & $<0,005$ & OS, $6 w=6, N-W$ plug-in \\
HML & 9,02 & 0 & $c=1, \mathrm{~L}=0,66$ \\
\hline$*$ Ancho de banda (bw), parámetros de truncamiento (m,cyL), kernel espectral cuadrático (OS), método \\
\multicolumn{2}{l}{ de estimación plug-in de Newey y West (N-W plug-in). } \\
\hline
\end{tabular}

Fuente: elaboración propia

De acuerdo con los resultados presentados en la tabla 2, la estimación del parámetro de diferenciación fraccional para el proceso de retornos del IGBC es 0,09, el cual, en apariencia, es pequeño. En términos de las pruebas, la mayoría de estas no encuentra suficiente evidencia como para rechazar la hipótesis nula de memoria corta, excepto la prueba RS de Lo, la cual presenta un valor $p$ inferior a 0,03 . Sin embargo, dado que la evidencia se inclina hacia el no rechazo de la hipótesis nula de memoria corta, se supondrá por ahora que el proceso de los retornos del IGBC no presenta indicios de memoria larga. Por otro lado, en términos de las transformaciones de los retornos, existe una fuerte evidencia hacia el rechazo de la hipótesis nula, lo que sugiere la posible presencia de memoria larga en el proceso de volatilidad condicional. 


\section{MODELO PROPUESTO PARA EL RETORNO DEL IGBC}

Spanos (1995) menciona que cualquier teoría relacionada con la $\mathrm{EMH}^{5}$ necesita tener en cuenta la evidencia empírica (hechos estilizados) encontrada en las series de tiempo financieras. En particular, se necesita considerar la memoria larga en la volatilidad condicional, la simetría y la leptocurtosis de la distribución detrás de los datos estudiados.

De acuerdo con Tsay (2005), en la mayoría de las series de retornos financieros las correlaciones seriales son débiles, y por tanto, construir una ecuación de media condicional es equivalente a remover la media muestral de los datos, siempre y cuando la media muestral sea significativamente diferente de cero. Este mismo autor, más adelante, menciona que para algunas series de retornos diarios, un simple modelo autorregresivo (AR) de orden uno podría ser adecuado.

Así, en este trabajo se plantea para el inicio de la modelación de los retornos del IGBC un modelo auto-regresivo de orden uno como consecuencia de la poca correlación encontrada en el análisis descriptivo anterior (ver gráfico 6). Por otro lado, y de acuerdo con la débil evidencia encontrada a favor de memoria larga en el proceso de media condicional, este componente es considerado junto con el esquema auto-regresivo para conformar un modelo auto-regresivo fraccionalmente integrado (ARFI(d)) como los planteados por Granger (1980), Granger y Joyeux (1980) y Hosking (1981). En cuanto a la volatilidad condicional, se considera un modelo HYAPARCH (1,1), de acuerdo con la evidencia de memoria larga presentada en el análisis descriptivo. Según Schoffer (2003) este modelo captura todos los hechos estilizados encontrados en la investigación empírica.

Para la estimación conjunta de este modelo, es usado el método de máxima verosimilitud condicional, donde la distribución condicional propuesta es la distribución de error generalizada (GED) (ver gráfico 2 y gráfico 3), equivalente a la propuesta por Nelson (1991). La estimación de los errores estándar se realiza mediante el método robusto, tal y como lo menciona Davidson (2011). Los resultados son presentados en la tabla 3.

Siglas en inglés del término hipótesis de eficiencia de mercado. 
Tabla 3. Modelo inicial: ARFI(d)-HYAPARCH(1,1)

\begin{tabular}{|c|c|c|c|c|}
\hline & Estimación & Err. Est. & Razón $t$ & Valor $p$ \\
\hline \multicolumn{5}{|c|}{ Modelo de media } \\
\hline GED nu & 1,28 & 0,08 & $\sim$ & $\sim$ \\
\hline ARFIMA d & 0,08 & 0,03 & 3,03 & 0,00 \\
\hline AR1 & 0,14 & 0,03 & 4,26 & 0,00 \\
\hline \multicolumn{5}{|c|}{ Modelo de volatilidad } \\
\hline Intercepto & 0,37 & 0,09 & $\sim \sim$ & $\sim$ \\
\hline Memoria & 0,25 & 0,11 & $-\sim$ & $\sim$ \\
\hline Amplitud & 0,38 & 0,19 & $\sim$ & $\sim$ \\
\hline Asimetría (mu) & 1,26 & 0,59 & 2,12 & 0,03 \\
\hline Potencia (eta) & 1,89 & 0,40 & - & - \\
\hline AR1 & 0,76 & 0,12 & 6,63 & 0,00 \\
\hline MAl & 0,68 & 0,12 & 5,44 & 0,00 \\
\hline \multicolumn{5}{|c|}{ Log Likelihood $=-3578,01$} \\
\hline \multicolumn{5}{|c|}{ Schwarz Criterion $=-3616,83$} \\
\hline \multicolumn{5}{|c|}{ Hannan-Quinn Criterion $=-3598,50$} \\
\hline \multicolumn{5}{|c|}{ Akaike Criterion $=-3588,01$} \\
\hline \multicolumn{5}{|c|}{ Ljung-Box (residuales est.): $\mathrm{Q}(30)=34,60\{0,26\}$} \\
\hline \multicolumn{5}{|c|}{ Ljung-Box (residuales est. al cuadrado): $Q(30)=180,04\{0\}$} \\
\hline
\end{tabular}

Fuente: elaboración propia

De acuerdo con los resultados presentados en la tabla 3, se observa que la estimación del parámetro $v$ de la distribución condicional GED es menor a 2, lo que sugiere una distribución condicional leptocúrtica. La estimación del parámetro de diferenciación fraccional $d$ resulta ser significativa, lo que indica que el proceso de media condicional de los retornos del IGBC presenta memoria larga. Además, se observa que el coeficiente auto-regresivo de orden uno resulta ser significativo a niveles convencionales de significancia, lo que evidencia también memoria corta en este mismo proceso. 
El modelo estimado inicial, por tanto toma la forma:

En media condicional,

$$
(1-\underbrace{0,14 L}_{\{0,03\}})(1-L)^{0,03} r_{t}^{0,03} r_{t}=a_{t} \text {, donde } a_{t}=\sigma_{t} \varepsilon_{t} .
$$

En volatilidad condicional,

$$
\begin{aligned}
& (1-\underbrace{0,68}_{0,12} L)\left(\sigma_{t}^{\{0,40} \sigma_{0,09}^{0,37}\right) \\
& {\left[(1-\underbrace{0,68 L}_{0,12} L)-(1-\underbrace{0,76}_{0,12} L)(1-\underbrace{0,38}_{0,19}\left((1-L) \frac{\{0,11\}}{0,25}-1\right))\right](1+\underbrace{1,26}_{0,59} S_{t})\left|a_{t}\right| \frac{\{0,40\}}{1,90},}
\end{aligned}
$$
mados.

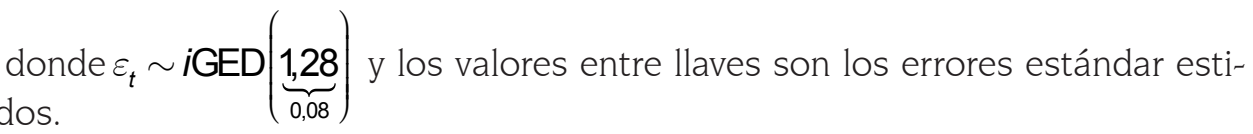

En términos generales, y al considerar la prueba de Ljung-Box para los residuales estandarizados hasta el rezago 30 , el modelo propuesto en media condicional se encuentra bien especificado, ya que captura de manera adecuada la estructura de correlación del proceso.

Por otro lado, al considerar el proceso de volatilidad condicional, la estimación del parámetro de memoria hiperbólica es $\hat{d}_{2} \approx 0,25$, lo que soporta la hipótesis de memoria larga en este proceso. La estimación del parámetro de amplitud es $\hat{\alpha} \approx 0,38$, el cual es menor a uno, lo que indica que el proceso es estacionario, tal como lo menciona Schoffer(2003). Además, la estimación del parámetro de asimetría $\hat{\mu} \approx 1,26$ es positiva y significativa, lo que evidencia que shocks positivos y negativos contribuyen de manera diferente a la volatilidad condicional; en este caso, debido a que la estimación de este efecto es positiva, la contribución a la volatilidad condicional de shocks negativos es mayor. Por otro lado, al considerar la estimación del parámetro de potencia asimétrica observada $\hat{\eta} \approx 1,89$, se encuentra que este está muy cercano a dos, lo que sugiere que la forma más adecuada para modelar este proceso es en términos de la varianza condicional. También se observa que la estimación de los parámetros GARCH del modelo son ambos estadísticamente significativos. Por último, se observa que la prueba de Ljung-Box para los residuales estandarizados al cuadrado hasta el rezago 30 indica que el modelo en el proceso de volatilidad condicional no alcanza a capturar toda la estructura de auto-correlación. Para examinar esta falta de especificación en el modelo, se procede a estimar la 
función de auto-correlación para los residuales estandarizados al cuadrado y realizar la prueba de heterocedasticidad condicional de McLeod-Li para los residuales estandarizados (ver gráfico 7).

Gráfico 7. (a) Función de autocorrelación estimada para los residuales estandarizados al cuadrado, y (b) prueba de McLeod-Li de heterocedasticidad condicional para los residuales estandarizados

a

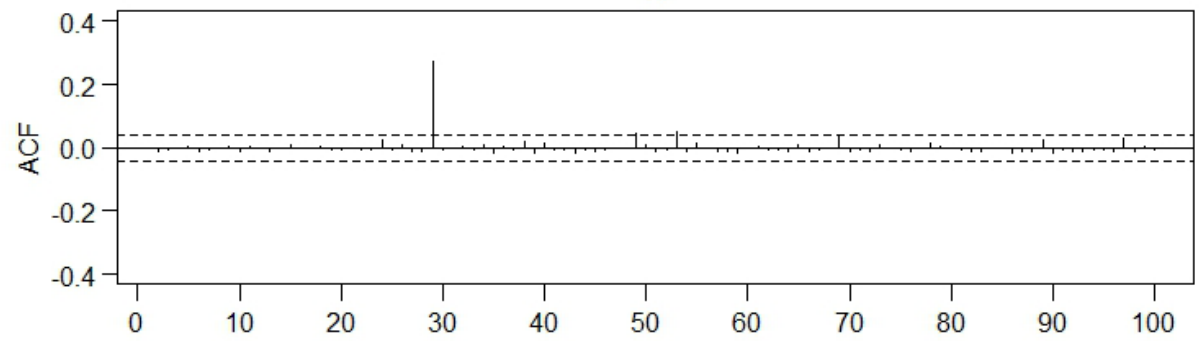

Rezago

$b$

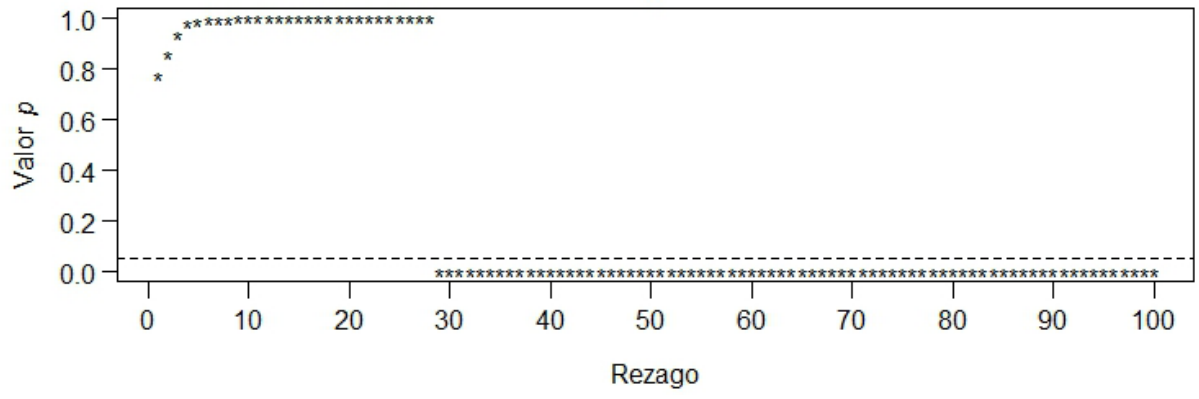

Fuente: elaboración propia

De acuerdo con el gráfico 7, todavía existe una auto-correlación estadísticamente significativa en el rezago 29. Al examinar los datos, se encuentra que esta auto-correlación se debe al retorno observado en el día 28 de diciembre de 2001 (ver gráfico 8). Este retorno se presenta muy cerca del inicio de la serie, y como consecuencia existe disponible muy poca información histórica para que el modelo pueda detectar este comportamiento. Debido a esto, se propone considerar este efecto mediante el uso de una variable dummy en el modelo de volatilidad condicional. Por otro lado, la estimación del parámetro de potencia en este proceso sugiere considerar la varianza condicional, por lo que se propone un modelo HY-A-GARCH, propuesto por Davidson (2004). 
Gráfico 8. Retorno observado en el día 28 de diciembre de 2001

$r_{t}$

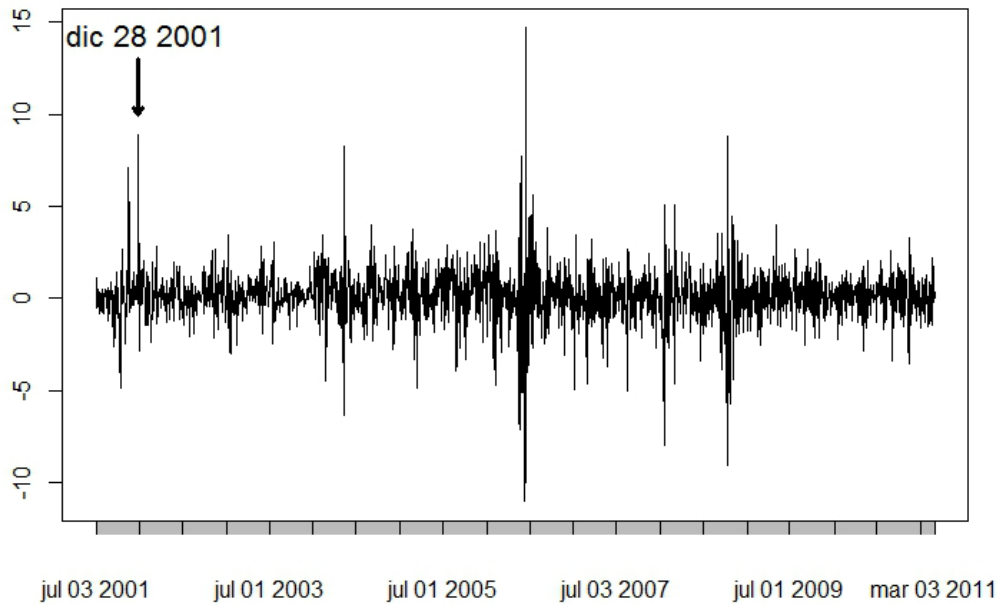

Fuente: elaboración propia

A continuación se presentan los resultados para el modelo ARFI-HYAGARCH:

Tabla 4. Modelo propuesto: ARFI(1,d)-HYAGARCH $(1,1)$

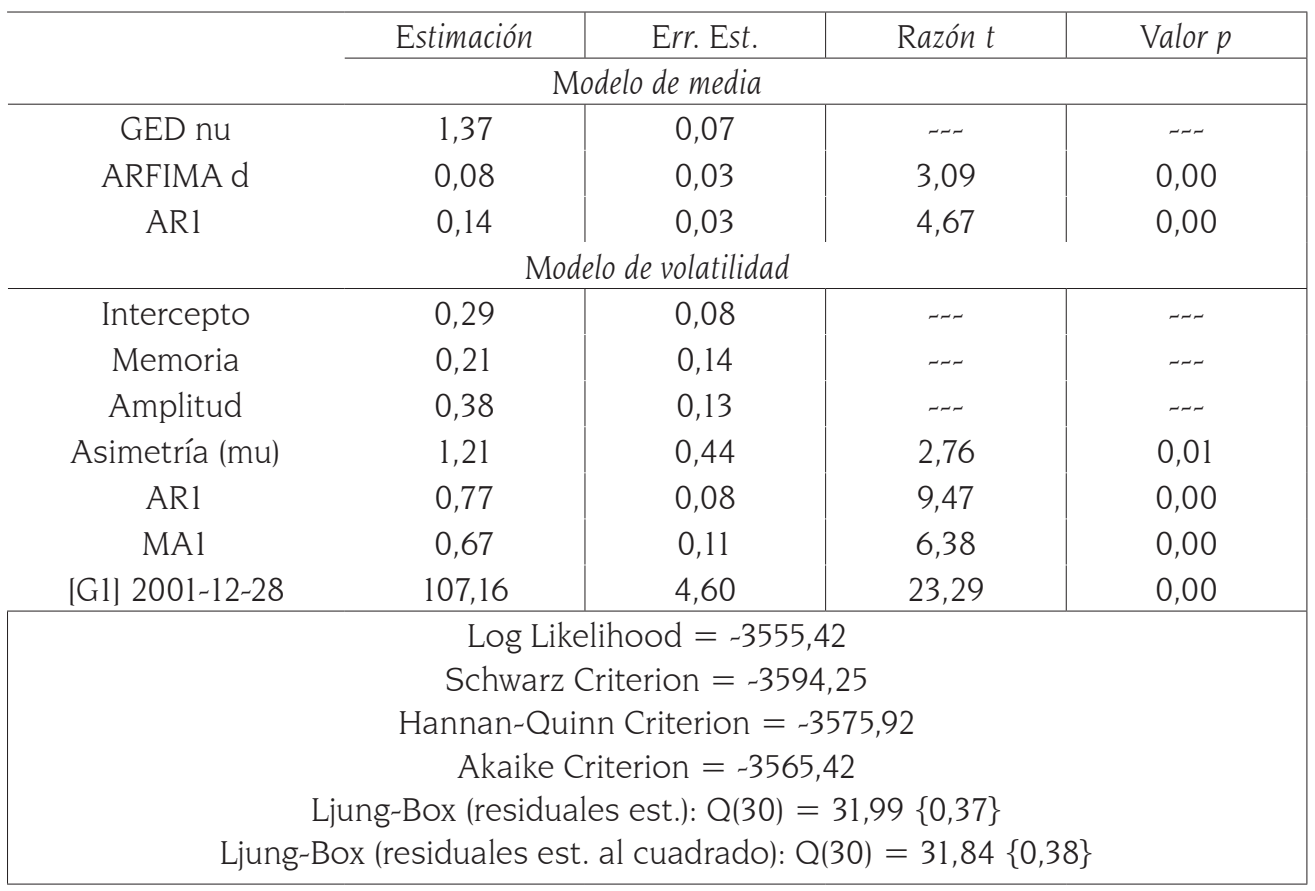

Fuente: elaboración propia 
Así, el modelo propuesto es de la forma:

En media condicional,

$$
(1-\underbrace{0,14 L}_{\{0,03\}})(1-\mathrm{L})_{0,0,08}^{\{0,03\}} r_{t}=a_{t}, \text { donde } a_{t}=\sigma_{t} \varepsilon_{t},
$$

En varianza condicional,

$$
\begin{aligned}
& (1-\underbrace{0,67}_{\{0,11\}} \mathrm{L})(\sigma_{t}^{2}-\underbrace{0,29}_{\{0,08\}}-\underbrace{107,16 \mathrm{G}_{01 / 12 / 28}}_{\{4,60\}})= \\
& \left.\left[(1-\underbrace{0,67 \mathrm{~L}}_{\{0,11\}})-(1-\underbrace{0,77}_{\{0,08\}} \mathrm{L})(1-\underbrace{0,38}_{\{0,13\}}(1-\mathrm{L})^{\frac{\{0,41}{0,21}}-1)\right)\right](1+\underbrace{1,21}_{\{0,44\}} S_{t}) a_{t}^{2},
\end{aligned}
$$
mados.

donde $\varepsilon_{t} \sim i \mathrm{GED}(\underbrace{1,37}_{\{0,07\}})$ y los valores entre llaves son los errores estándar esti-

De acuerdo con la tabla 4, y al considerar los criterios de información de Schwarz (1978), Rissanen (1978), Hannan y Quinn (1979), Akaike (1973, 1974), el modelo propuesto parece ser mejor con respecto al modelo considerado de manera inicial (ver tabla 3), y en general las estimaciones no varían de manera considerable con respecto al modelo inicial. Por otro lado, y de acuerdo con la prueba de Ljung-Box para los residuales estandarizados al cuadrado, existen indicios de que el modelo propuesto en el proceso de volatilidad es adeado al capturar la estructura de auto-correlación. En el gráfico 9 se presentan las funciones de auto-correlación estimadas para los residuales estandarizados y sus transformaciones.

Al observar las funciones de auto-correlación estimadas para los residuales estandarizados y sus transformaciones, presentadas en el gráfico 9, se evidencia que el modelo propuesto parece capturar de manera adecuada toda la estructura de auto-correlación del proceso de retornos del IGBC, tanto en media como en varianza condicional. Sin embargo, a simple vista pareciera que algunas autocorrelaciones para los residuales estandarizados y los residuales estandarizados al cuadrado fueran significativas. Para evaluar esta significancia, en el gráfico 10 se presentan las pruebas de Ljung-Box y McLeod-Li para los residuales estandarizados. De acuerdo con el gráfico, se puede observar que ambas pruebas indican que las auto-correlaciones estimadas, tanto para los residuales estandarizados como para los residuales estandarizados al cuadrado, en conjunto, no son estadísticamente 
significativas hasta el rezago cien. Esto refuerza el hecho de que el modelo propuesto captura de manera adecuada toda la estructura de auto-correlación del proceso de retornos del IGBC.

Gráfico 9. (a) Función de autocorrelación para los residuales estandarizados, (b) función de autocorrelación para los residuales estandarizados al cuadrado, y (c) función de autocorrelación para los valores absolutos de los residuales estandarizados

(a)

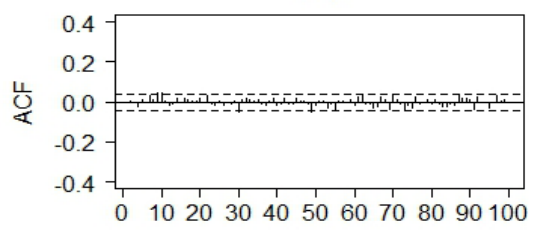

Rezago (b)

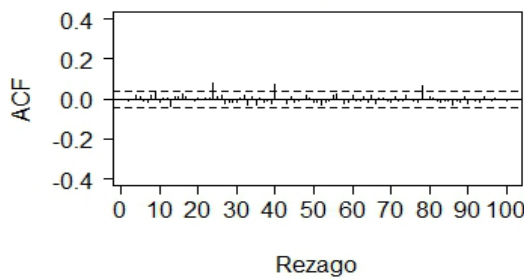

(c)

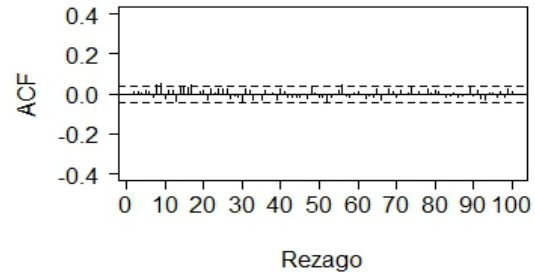

Fuente: elaboración propia

El gráfico 11 presenta los residuales del modelo estimado. En el panel (a), se observa que la distribución de los residuales estandarizados es simétrica alrededor de cero y es menos leptocúrtica que la distribución de los datos originales (retornos del IGBC). Además, y de acuerdo con Hsieh (1989), cuando la varianza condicional se encuentra especificada de manera correcta, el exceso de curtosis en los residuales estandarizados no puede exceder el exceso de curtosis en los residuales no estandarizados. Para este caso, la estimación del exceso de curtosis presentado en los residuales no estandarizados es igual a 12,72, que comparado con el exceso de curtosis para los residuales estandarizados igual a 2,77, indica que el modelo se encuentra bien especificado. Por otro lado, y de acuerdo con el panel (b), la gran mayoría de los residuales estandarizados se encuentra dentro de dos errores estándar, y muestran una gran homogeneidad, aparte del residual estandarizado presentado al inicio de la serie 6 . También, se observa que el modelo propuesto

6 Este residual se presenta exactamente el 16 de noviembre de 2001, fecha muy similar a la observación considerada anteriormente. Este comportamiento, nuevamente se debe a que la observación se encuentra muy cercana del inicio de la serie, donde no existe mucha información histórica del proceso, lo que hace que el modelo sea incapaz de capturar el comportamiento para esta fecha. 
captura de manera adecuada la dependencia de segundo orden observado en el proceso de retornos del IGBC; el hecho estilizado de volatilidad por agrupamiento ya no se encuentra presente.

Gráfico 10. (a) Prueba de Ljung-Box para los residuales estandarizados, y (b) prueba de McLeod-Li para los residuales estandarizados. Ambas pruebas se realizan hasta el rezago cien

(a)

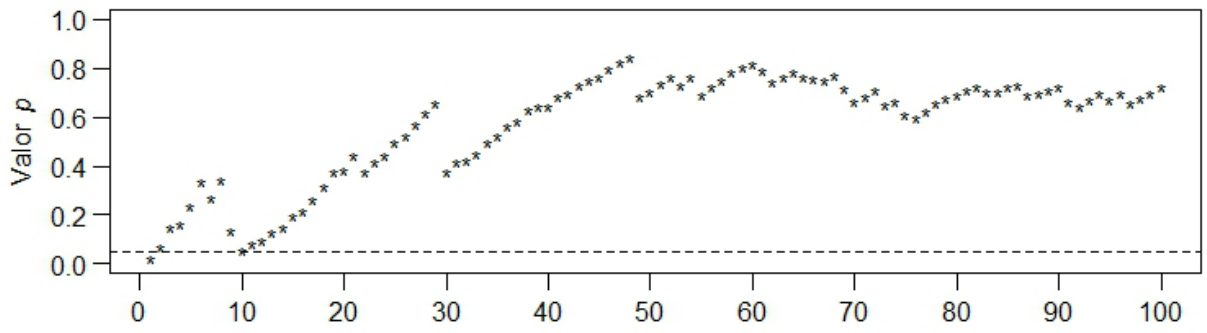

(b)

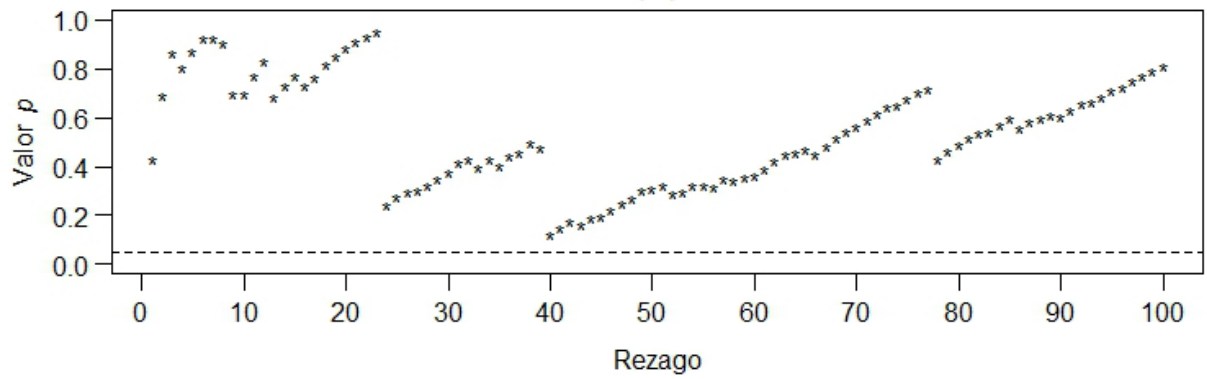

Fuente: elaboración propia

Adicional al análisis anterior, a continuación se prueba mediante el test BDS de Brocky otros (1996) si aún existe evidencia de relaciones no lineales en los residuales estandarizados. Los resultados para esta prueba son presentados en la tabla 5.

Tabla 5. Prueba BDS para los residuales estandarizados. Las celdas de la tabla representan los valores $p$ para distintos valores de $\boldsymbol{m}$ y $\epsilon$

\begin{tabular}{|c|c|c|c|c|}
\hline \multirow{2}{*}{$m^{*}$} & \multicolumn{4}{|c|}{ o para puntos de cierre } \\
& 0,50 & 1,00 & 1,51 & 2,01 \\
\hline 2 & 0,61 & 0,95 & 0,72 & 0,38 \\
\hline 3 & 0,40 & 0,66 & 0,94 & 0,45 \\
\hline 4 & 0,36 & 0,51 & 0,84 & 0,63 \\
\hline
\end{tabular}


Prueba de eficiencia débil en el mercado accionario colombiano

\begin{tabular}{|c|c|c|c|c|}
\hline \multirow{2}{*}{$m^{*}$} & \multicolumn{4}{|c|}{ O para puntos de cierre } \\
& 0,50 & 1,00 & 1,51 & 2,01 \\
\hline 5 & 0,35 & 0,42 & 0,75 & 0,79 \\
\hline
\end{tabular}

*Dimensión de integración

Fuente: elaboración propia

Gráfico 11. (a) Histograma de los residuales estandarizados con densidad kernel estimada (línea continua) y densidad bajo la hipótesis de normalidad (línea punteada). (b) Serie de residuales estandarizados con intervalo de variabilidad ( \pm 2 errores estándar)

(a)

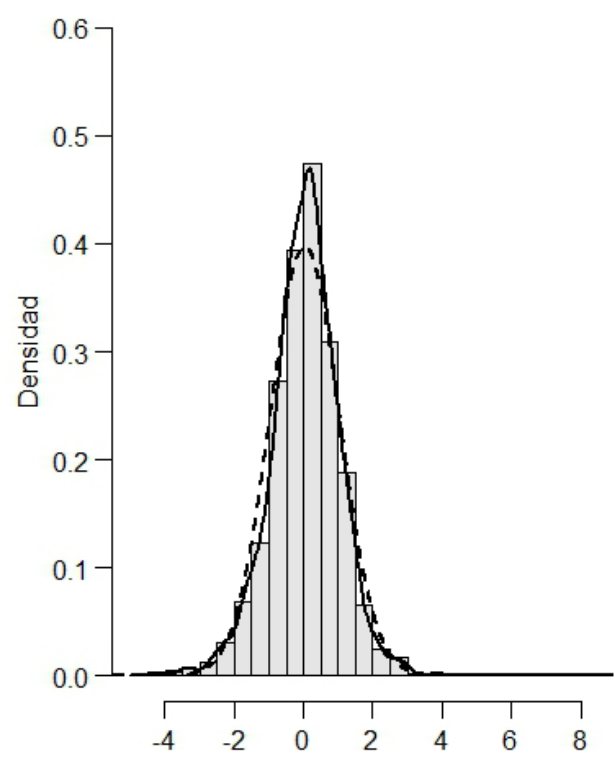

(b)

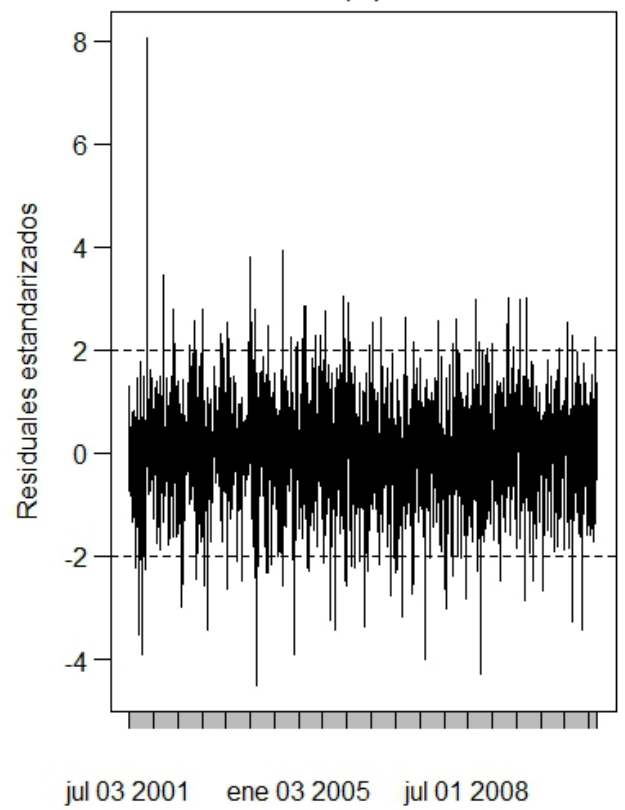

Fuente: elaboración propia

En la tabla 5 se observa cómo para diferentes dimensiones de integración, y para diferentes puntos de cierre, la prueba BDS no encuentra evidencia suficiente como para rechazar la hipótesis nula de que los errores estandarizados son variables aleatorias independientes e idénticamente distribuidas, lo que consolida el hecho de que el modelo propuesto es adecuado.

En el gráfico 12 se puede observar el buen desempeño del modelo estimado, ya que al considerar la estimación del proceso de media condicional, la volatilidad condicional exhibida en los residuales no estandarizados queda estimada de manera 
adecuada (ver panel c). Cabe resaltar que el modelo propuesto también captura de manera correcta los eventos de mayor impacto histórico en el IGBC.

En resumen, el modelo propuesto, en términos de la media condicional, indica la existencia de dos posibles fuentes de predictibilidad. Por un lado, la existencia de memoria corta con la presencia de una componente auto-regresiva de orden uno, y por el otro, la existencia de memoria a largo plazo. De esta forma, la hipótesis de martingala en diferencias en los retornos del IGBC se rechaza, y por lo tanto también la hipótesis de eficiencia del mercado en su forma débil. Cabe señalar que en este caso esta hipótesis es rechazada bajo el supuesto de que no existen costos de transacción, también que por parte de los participantes del mercado existe una neutralidad hacia el riesgo, y que la información del mercado puede ser obtenida sin ningún costo.

Gráfico 12. (a) Estimación del proceso de media condicional $\mu_{t}$, (b) varianza condicional estimada $\hat{\sigma}_{t}^{2}$, y (c) residuales no estandarizados con bandas de variabilidad $\left(\hat{a}_{t} \pm 2 \hat{\sigma}_{t}\right)$

(a)

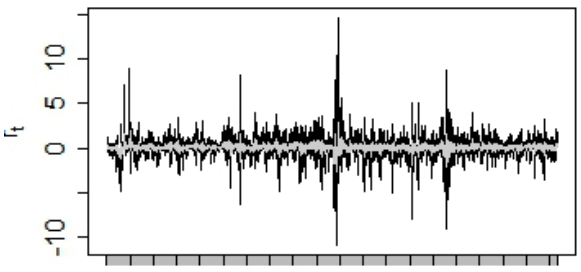

jul 032001 ene 032005 jul 012008 (b)

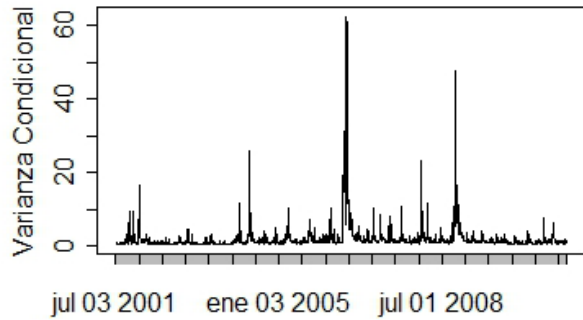

(c)

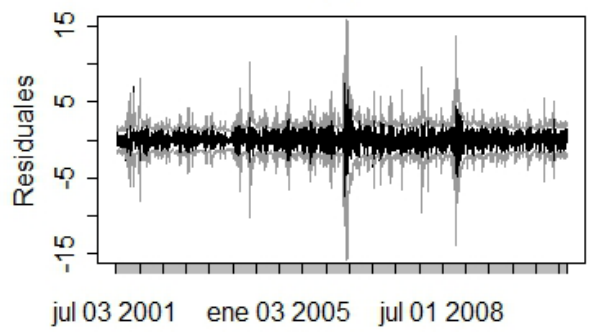

Fuente: elaboración propia

\section{CONCLUSIONES Y RECOMENDACIONES}

En este trabajo, se consideró la modelación de los retornos del IGBC. El proceso generador de estos parece obedecer, en media condicional, a un proceso autorregresivo de orden uno con una estructura de dependencia a largo plazo. Esto índica 
que la hipótesis de martingala en diferencias, y por tanto, la hipótesis de eficiencia débil no se cumplen. En cuanto a la varianza condicional, el proceso obedece a un modelo hiperbólico-asimétrico-generalizado autorregresivo condicionalmente heterocedástico (HYAGARCH), el cual considera una estructura de dependencia a largo plazo, además del efecto de asimetría (leverage) encontrados en las series financieras.

Dado que la hipótesis de eficiencia de mercado constituye la piedra angular de la teoría cuantitativa de mercados de capitales, es poco probable un rechazo inmediato y absoluto de la teoría. De esta manera, y de acuerdo con Spanos (1995), la experiencia en la modelación econométrica sugiere que es necesario más evidencia confiable que dé soporte a los resultados obtenidos, ya sea dentro del mercado colombiano (al comprobar la hipótesis también para los diferentes títulos que conforman el mercado) o en mercados emergentes similares (por ejemplo, los latinoamericanos). También es importante considerar el efecto que pueda tener los pocos años de existencia de la Bolsa de Valores de Colombia mediante factores como el tamaño de muestra, el número de sociedades cotizadas, la débil dispersión del capital, entre otros. Además, hay que considerar que en este trabajo, la hipótesis débil de eficiencia de mercado es rechazada bajo el supuesto que no existen costos de transacción, que por parte de los participantes del mercado existe una neutralidad hacia el riesgo, y que la información del mercado puede ser obtenida sin ningún costo. Por tanto, sería interesante plantear una metodología que incluya estos aspectos y así mejorar este tipo de investigaciones.

Por otro lado, en este trabajo se emplean solo métodos paramétricos para la modelación de los retornos. Sería también interesante utilizar métodos más flexibles que permitan capturar dináminas más generales como los semiparamétricos propuestos por Engle y González-Rivera (1991) y los no paramétricos como los de Fan y Yao (2005) para efectos de la modelación.

\section{BIBLIOGRAFÍA}

Akaike, H. (1973). Information Theory and An Extension of the Maximum Likelihood Principle. En: B. N. Petrov, F. Csaki, B. N. Petrov, y F. Csaki (Edits.), 2nd International Symposium on Information Theory. Budapest, AkademiaiKiado, pp. 267-281.

Akaike, H. (1974). A New Look at the Statistical Model Identification. En: IEEE Transactions on Automatic Control, Vol. 19, N. ${ }^{\circ}$ 6, pp. 716-723.

Andreou, E.,Pittis, N. ySpanos, A. (2001). On Modelling Speculative Prices: The Empirical Literature.En: Journal of Economic Surveys, Vol. 15, N. ${ }^{\circ}$ 2, pp. 187-220. 
Baillie, R. T. (1996). Long Memory Processes and Fractional Integration in Econometrics. En: Journal of Econometrics (Elsevier, Ed.), Vol. 73, N. ${ }^{\circ}$ 1, 5-59.

Baquero Herrera, M. (2006). El Observatorio Financiero. Bogotá, Universidad Externado de Colombia, Departamento de Derecho Económico, 49 pp.

Bowman, A. W. yAzzalini, A. (1997). Applied Smoothing Techniques for Data Analysis: The Kernel Approach With S-Plus Illustrations. New York, Oxford University Press, 208 pp.

Bowman, A. W. yAzzalini, A. (2010). R package sm: nonparametric smoothing methods.R package version 2 , pp. 2-4.

Brock, W. A.; Dechert, W. D.; LeBaron, B. yScheinkman, J. A. (1996). A Test for Independence Based on the Correlation Dimension.En: Econometric Reviews, Vol. 15, N. ${ }^{\circ}$ 3, pp. 197-235.

Campbell, J. Y.; Lo, A. W. y MacKinlay, A. C. (1997). The Econometrics of Financial Markets. Princeton, New Jersey, Princeton University Press, 632 pp.

Chan, K.y Ripley, B. (2012). TSA: Time Series Analysis. R package version 1.0.

Davidson, J. (2004). Moment and Memory Properties of Linear Conditional Heteroscedasticity Models, and a New Model.En: Journal of Business and Economic Statistics, Vol. 22, N. ${ }^{\circ}$, pp. 16-29.

Davidson, J. (2011). Time Series Modelling, Version 4.33. Exeter, University of Exeter, The Business School, 77 pp.

Dimson, E. yMussavian, M. (2000). Market Efficiency.En: The Current State of Business Disciplines, Vol. 3, N. ${ }^{\circ}$, pp. 959-970.

Ding, Z. y Granger, C. W. (1993). A Long Memory Property of Stock Market Returns and a New Model. En:Journal of Empirical Finance, Vol. 1, N. ${ }^{\circ}$ 1, pp. 86-106.

Ding, Z.; Granger, C. W. y Engle, R. F. (1996). Modeling Volatility Persistence of Speculative Returns: A New Approach.En: Journal of Econometrics, Vol. 73, N. ${ }^{\circ}$ 1, pp. 185-215.

Doornik, J. A. (2007). Object-Oriented Matrix Programming Using Ox (3ra Ed.). London, Timberlake Consultants Press and Oxford, 376 pp.

Engle, R. (1982). Autoregressive Conditional Heteroscedasticity with Estimates of the Variance of United Kingdom Inflation.En:Econometrica, Vol. 50, N. 4, pp. 987-1007.

Engle, R. F. y González-Rivera, G. (1991). Semiparametric ARCH Models. En:Journal of Business y Economic Statistics, Vol. 9, N. ${ }^{\circ} 4$, pp. 345-359.

Escanciano, J. C. y Lobato, I. N. (2009). Testing the Martingale Hypothesis. En: T. C. Mills, y K. Patterson (Edits.), Palgrave Handbook of Econometrics, Vol. 2: Applied Econometrics. New York, Palgrave MacMillan, pp. 972-1003.

Fama, E. F. (1970). Efficient Capital Markets: A Review of Theory and Empirical Work.En: The Journal of Finance, Vol. 25, N. ${ }^{\circ}$ 2, pp. 383-417.

Fan, J. y Yao, Q. (2005). Nonlinear Time Series: Nonparametric and Parametric Methods (1ra Ed.). New York, Springer-Verlag, 552 pp. 
Genz, A., y Bretz F. (2009). Computation of Multivariate Normal and t Probabilities, Lecture Notes in Statistics. Berlin Heidelberg, Springer-Verlag, Vol. 195, 126 pp.

Genz, A.;Bretz, F.;Miwa, T.;Mi, X.;Leisch, F.;Scheipl, F. y Hothorn, T. (2012). mvtnorm: Multivariate Normal. R package version 0.9-9993.

Gil-Alana, L. A. (2003). Testing the Power of a Generalization of the KPSS-Tests against Fractionally Integrated Hypotheses.En:ComputationalEconomics, Vol. 22, N. ${ }^{\circ}$ 1, pp. 23-38.

Granger, C. W. (1980). Long Memory Relationships and the Aggregation of Dynamic Models.En: Journal of Econometrics, Vol. 14, N. ${ }^{\circ}$ 2, pp. 227-238.

Granger, C. W. yJoyeux, R. (1980). An Introduction to Long Memory Time Series Models and Fractional Differencing. En:Journal of Time Series Analysis, Vol. 1, N.․ 2, pp. 15-39.

Graves, S. (2012). FinTS: Companion to Tsay (2005) Analysis of Financial Time Series. R packageversion $0.4-4$.

Hannan, E. J. yQuinn, B. G. (1979). The Determination of the Order of an Autoregression. En Journal of the Royal Statistical Society. Series B (Methodological), Vol. 41, N. 2, pp. 190-195.

Harris, D.; McCabe, B. yLeybourne, S. (2008). Testing for Long Memory. En:Econometric Theory, Vol. 24, N. ${ }^{\circ} 1,143-175$.

Hosking, J. R. (1981). Fractional Differencing. En:Biometrika, Vol. 68, N. ${ }^{\circ}$ 1, pp. 165-176.

Hsieh, D. A. (1989). Modelling Heteroscedasticity in Daily Foreign-Exchange Rates. En:Journal of Business y Economic Statistics, Vol. 7, N. ${ }^{\circ}$ 3, pp. 307-317.

Jarque, C. M. yBera, A. K. (1987). A Test for Normality of Observations and Regression Residuals. En:International Statistical Review, Vol. 55, N.․ 2, pp. 163-172.

Lee, D. y Schmidt, P. (1996). On the Power of the KPSS Test of StationarityAgainst FractionallyIntegrated Alternatives. En: Journal of Econometrics, Vol. 73, N. ${ }^{\circ}$ 1, pp. 285-302.

LeRoy, S. F. (1989). Efficient Capital Markets and Martingales. En:Journal of Economic Literature, Vol. 27, N. ${ }^{\circ} 4$, pp. 1583-1621.

Ljung, G. M. y Box, G. E. (1978). On a Measure of Lack of Fit in Time Series Models. En:Biometrika, Vol. 65, N.․ 2, pp. 297-303.

Lo, A. W. (1991). Long-Term Memory in Stock Market Prices. En:Econometrica, Vol. 59, N. 5, pp. 1279-1313.

Mandelbrot, B. B. (1966). Forecasts of Future Prices, Unbiased Markets, and "Martingale" Models. En: Journal of Business, Vol. 39, N. ${ }^{\circ}$ 1, pp. 242-255.

Mandelbrot, B. B. (1971). When Can Price be Arbitraged Efficiently? A Limit to the Validity of the Random Walk and Martingale Models. En: The Review of Economics and Statistics, Vol. 53, N. 3, pp. 225-236.

Maya Ochoa, C. y Torres Avendaño, G. I. (2004). The Unification of the Colombian Stock Market: A Step Towards Efficiency-Empirical Evidence.En:Latin American Business Review, Vol. 5, N. .4 , pp. 69-98. 
Maya Ochoa, C. y Torres Avendaño, G. I. (2005). Las caminatas aleatorias no son de este mundo. Teoría y revisión bibliográfica sobre evidencia empírica. En: Revista Universidad EAFIT, Vol. 41, N. ${ }^{\circ} 138$, pp. 65-83.

McLeod, A. I. y Li, W. K. (1983). Diagnostic Checking ARMA Time Series Models Using SquaredResidual Autocorrelations. En: Journal of Time Series Analysis, Vol. 4, N. ․ 4, pp. 269-273.

McLeod, A. I. y Zhang, Y. (2008). Improved Subset Autoregression: With R Package. En:Journal of Statistical Software, Vol. 28, N. $^{\circ}$ 2, pp. 1-28.

Méndez Chaves, A. (2009). Revisión a la Eficiencia del Mercado de Capitales Colombiano. Bogotá, Universidad Nacional de Colombia, Sede Bogotá, Facultad de Ciencias Económicas, Escuela de Economía, $31 \mathrm{pp}$.

Montenegro García, A. (2007). El Efecto Día en la Bolsa de Valores de Colombia. Bogotá, Pontificia Universidad Javeriana, Facultad de Ciencias Económicas y Administrativas, Departamento de Economía, 21 pp.

Nelson, D. B. (1991). Conditional Heteroskedasticity in Asset Returns: A New Approach. En:Econometrica, Vol. 59, N. ${ }^{\circ}$ 2, pp. 347-370.

Newey, W. K. y West, K. D. (1987). A Simple, Positive Semi-definite, Heteroskedasticity and Autocorrelation Consistent Covariance Matrix. En:Econometrica, Vol. 55, N. ${ }^{\circ}$ 3, pp. 703-08.

Newey, W. K. y West, K. D. (1994). Automatic Lag Selection in Covariance Matrix Estimation. En:Review of Economic Studies, Vol. 61, N.² 4, pp. 631-653.

Ocampo, J. A. (2009). Latin America and the Global Financial Crisis.En:Cambridge Journal of Economics, Vol. 33, N. ${ }^{\circ} 4$, pp. $703-724$.

Pérez Pérez, J. E. (2009). An Assessment of Long Memory Properties of Stock Returns in Colombia's Stock Market. Bogotá, Universidad del Rosario, Facultad de Economía, 23 pp.

Pérez Ramírez, F. O. y Fernández Castaño, H. (2006). Análisis de la volatilidad del Índice General de la Bolsa de Valores de Colombia utilizando modelos ARCH. En:Revista Ingenierías Universidad de Medellín, Vol. 5, N. ${ }^{\circ}$, pp. 13-33.

R Core Team. (2012). R: A language and environment for statistical. Vienna, Austria: R Foundation for Statistical Computing. ISBN 3-900051-07-0.

Rissanen, J. (1978). Modeling by Shortest Data Description.En:Automatica, Vol. 14, N. ${ }^{\circ}$, pp. $465-471$.

Rivera Palacio, D. M. (2009). Modelación del Efecto Día de la Semana para los Índices Accionarios de Colombia. En: Revista de Economía del Rosario, Vol. 12, N. 1, pp. 1-24.

Roberts, H. V. (1967). Statistical versus Clinical Prediction of the Stock Market. (U. o. Chicago, Ed.) Unpublished manuscript.

Robinson, P. M. (1995). Log-Periodogram Regression of Time Series with Long Range Dependence. En:The Annals of Statistics, Vol. 23, N. ${ }^{\circ} 3$, pp. 1048-1072. 
Robinson, P. M. y Lobato, I. N. (1998). A Nonparametric Test fosI(0).En: The Review of Economic Studies, Vol. 65, N. ${ }^{\circ}$ 3, pp. 475-495.

Ryan, J. A. y Ulrich, J. M. (2012). xts: eXtensible Time Series. R package version 0.8 8.

Samuelson, P. A. (1965). Proof that Properly Anticipated Prices Fluctuate Randomly. En: Industrial Management Review, Vol. 6, pp. 41-49.

Schoffer, O. (2003). HY-A-PARCH: A Stationary A-PARCH Model with Long Memory. Dortmund, Universität Dortmund, D-44221, Fachbereich Statistik, 14 pp.

Schwarz, G. (1978). Estimating the Dimension of a Model. En: The Annals of Statistics, Vol. 6, N. 2 , pp. 461-464.

Silverman, B. W. (1986). Density Estimation for Statistics and Data Analysis (1ra Ed.). Londres, Chapman and Hall, 176 pp.

Spanos, A. (1995). On Theory Testing in Econometrics: Modeling with Nonexperimental Data. En:Journal of Econometrics, Vol. 67, N. ${ }^{\circ}$ 1, pp. 189-226.

Trapletti, A. yHornik, K. (2012). tseries: Time Series Analysis and Computational Finance. R package version 0.10-29.

Tsay, R. S. (2005). Analysis of Financial Time Series (2da Ed.). New Jersey, John Wiley y Sons, 712 pp.

Willinger, W.;aqqu, M. S. yTeverovsky, V. (1999). Stock Market Prices and Long-Range Dependence. En:Finance and Stochast, Vol. 3, N. ${ }^{\circ}$ 1, pp. 1-13.

Worthington, A. C. y Higgs, H. (2003). Tests of Random Walks and Market Efficiency in Latin American Stock Markets: An Empirical Note. Brisbane, Queensland University of Technology, School of Economics and Finance, 14 pp. 


\section{ANEXOS}

Anexo A. Paquetes de R utilizados en las estimaciones

\begin{tabular}{|l|l|l|}
\hline \multicolumn{1}{|c|}{ Paquete } & \multicolumn{1}{c|}{ Uso } & \multicolumn{1}{c|}{ Referencia } \\
\hline xts & Manejo del formato de las fechas & Ryan y Ulrich (2012) \\
\hline sm & Estimación de densidades bivariadas y contornos & Bowman y Azzalini (2010) \\
\hline mvtnorm & Generación de variables aleatorias bivariadas & Genz y otros (2012) \\
\hline FinTS & Estimación de la función de Autocorrelación & Graves (2012) \\
\hline TSA & $\begin{array}{l}\text { Prueba de McLeod-Li y cálculo de los coeficien- } \\
\text { tes de curtosis y de asimetría }\end{array}$ & Chan y Ripley (2012) \\
\hline FitAR & Prueba de Ljung-Box & McLeod y Zhang (2008) \\
\hline tseries & Prueba de Jarque-Bera y la BDS & Trapletti y Hornik (2012) \\
\hline
\end{tabular}

Fuente: elaboración propia 